\title{
Semantic Exploration of DNS
}

\author{
Samuel Marchal, Jérôme François, Cynthia Wagner, and Thomas Engel \\ SnT - University of Luxembourg, Luxembourg \\ firstname.lastame@uni.lu
}

\begin{abstract}
The DNS structure discloses useful information about the organization and the operation of an enterprise network, which can be used for designing attacks as well as monitoring domains supporting malicious activities. Thus, this paper introduces a new method for exploring the DNS domains. Although our previous work described a tool to generate existing DNS names accurately in order to probe a domain automatically, the approach is extended by leveraging semantic analysis of domain names. In particular, the semantic distributional similarity and relatedness of sub-domains are considered as well as sequential patterns. The evaluation shows that the discovery is highly improved while the overhead remains low, comparing with non semantic DNS probing tools including ours and others.
\end{abstract}

\section{Introduction}

DNS (Domain Name System) [15] is critical for the well functioning of Internet as it is mainly used for locating a host in the Internet based on a human readable name. Service availability is improved by dynamic reallocation to another machine without changing the DNS name. However, this mechanism is also employed by attackers to improve the robustness and the efficiency of the attacks [18. Hence, DNS has recently gained interest from the security community and especially the naming scheme for discovering malware hosting domains 18 .

This paper focuses on DNS probing, i.e. guessing domains that are in use. This is an alternative to IP address scanning, which is fastidious and quite visible whereas DNS requests go through intermediate DNS servers, which hide the attackers. An attacker commonly uses dictionaries to probe existing domain names and aims to discover the networking organization, as well as potential vulnerable hosts. A common example is to check the hostnames of common services like FTP (File Transfer Protocol) or SSH (Secure Shell) by probing domains such as ftp. example.com. In [10, the authors show that DNS scanning allow to identify potentially vulnerable IPv6 addresses quicker than with a classical random IP scanning due to the large address space, they apply this technique to ease the spread of worms in IPv6 Internet.

Thus, penetration testing and security assessment are based on an initial recon by discovering subdomains and hosts. With a proper DNS configuration, this cannot be gathered directly and therefore requires brute-forcing. In this paper, the DNS brute forcing tool is semantically extended since we have observed that human based names usually follow semantic schemes. This includes the word semantic as well as numerical semantics (series of numbers) of DNS names.

R. Bestak et al. (Eds.): NETWORKING 2012, Part I, LNCS 7289, pp. $370-384,2012$.

(C) IFIP International Federation for Information Processing 2012 
The paper is organized as follows. Section 2 presents DNS. An overview of the semantic exploration system and SDBF (Smart DNS Brute-Forcer) [21] is given in section 3. Semantic extensions are covered in section 4. Our approach is assessed in section 5. Related work is presented in section 6] and conclusions are drawn in section 7

\section{DNS Background}

To keep the paper self-contained, this is a short overview of DNS, but the reader may read [16 1517] for further explanation.

The main objective of DNS is to provide a map between human readable and remainable names to IP addresses. The organization of DNS is hierarchical with a root server at the top and dedicated authoritative servers for each subdomain. Assuming the domain name www. uni. $1 \mathrm{u}, \mathrm{lu}$ is the top level domain (TLD) which is the parent of all . $\mathrm{lu}$ subdomains (second level domain) including uni.lu. The third level domain is www . uni . lu. When a user needs the IP address of www . uni . lu, the first step is to query a recursive DNS server, usually maintained by his operator. This server is responsible to find the host by iteratively querying the authoritative servers of the subdomains. So, it starts by asking a root server which replies back with the DNS server in charge of the $1 u$ domain. The recursive DNS server of the client can also contact it to know, which server is in charge of uni. lu. Finally, when uni . $l u$ is queried, it returns the IP address of www . uni . lu, which is then forwarded to the client by the recursive server.

DNS messages are mainly composed of Resource Records (RRs), which refers to different types of resolution. For the most common one the type is A or AAAA for getting the IPv4 or IPv6 address corresponding to a domain name. PTR are defined to enable inverse resolution (IP address to name).

A DNS name uses a dotted format to separate several components, i.e. a sequence of labels. In this paper, label $_{i}$ refers to the $i^{\text {th }}$ component, starting from the right. Thus, the top level domain is defined by label $_{0}$. For example, www.uni. $\mathrm{lu}$ has three labels: label $_{0}=$ lu, label $_{1}=u n i$, label $_{2}=w w w$. Even if a recent extension allows non-ASCII characters [7, this paper doesn't consider them, as most of domains are still composed only of ASCII characters.

\section{Exploration of DNS}

\subsection{System Overview}

Our approach aims to automatically discover DNS names, and in particular, some subdomains of a domain by generating labels. Assuming a domain $d$, most of the current techniques rely on testing labels sequentially, $l$, stored in a dictionary (www, ns, ftp, smtp, etc. but also atlanta, boston, host, etc.) by concatenating the label $l$ with the domain $d$ to form a new subdomain $l$.d. In this paper, our prior tool SDBF 21] is used to generate new names after a learning stage. Samples are required to learn how valid labels of domain names look like. They are collected through a passive DNS platform [22, which consists in monitoring 


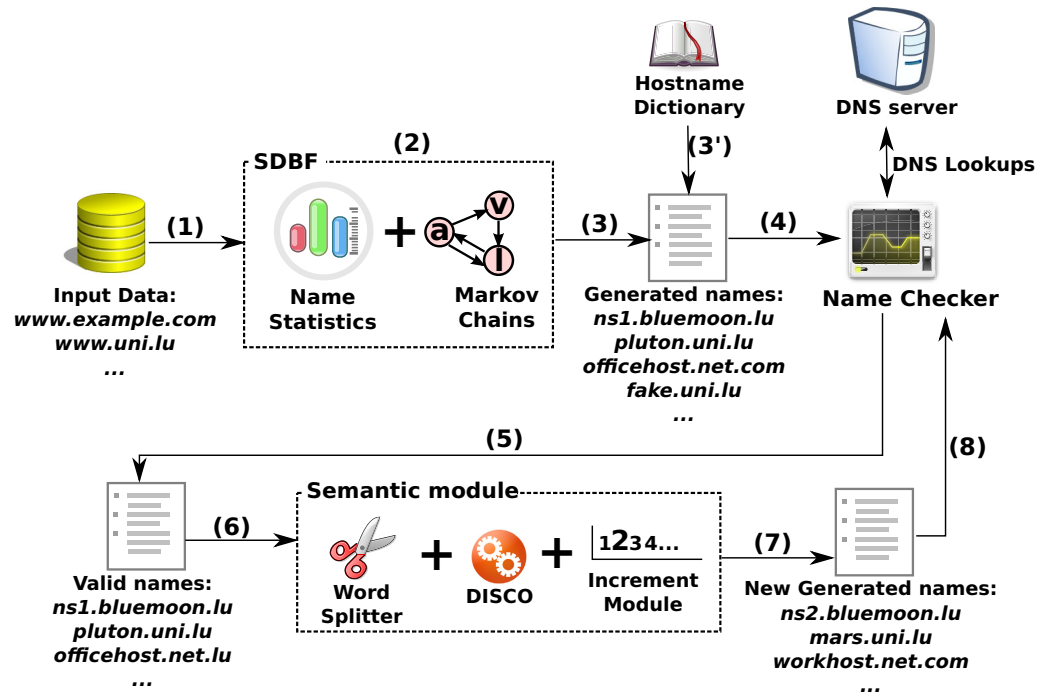

Fig. 1. System overview

and storing requests sent and replies received by a recursive DNS server. In our case, only valid names i.e. with valid DNS replies, are stored in a database.

Two key ideas have emerged from observations we made during our personal experience, as well as by mining the passive DNS database:

- subdomains of the same domain are semantically related, in particular end hosts. For example, using planets, cities, countries, or character names of cartoons is a frequent habit of network administrators,

- a domain may present sequential patterns. For example, enumeration is a standard naming convention within a company or a university that leads to hostnames like room1-pc1, room1-pc2, room2-pc1, ns1, ns2, etc.

As shown in figure 1 the two main steps for discovering the DNS names are:

- the construction of an initial list of names using SDBF [21] (2) or a dictionary based-approach (3')

- the extension of that list by exploiting the semantics of names (5)-(8)

\subsection{SDBF}

Features. The main features in SDBF are based on linguistic parameters. We assume an input list (1) of DNS names $N=\left\{n_{1}, \ldots, n_{P}\right\}$, a set of DNS label levels $L=\left\{l_{1}, \ldots, l_{S}\right\}$, a set of used characters $C=\left\{c_{1}, \ldots, c_{M}\right\}$ and a set of n-grams, $G_{x}=\left\{x_{1}, \ldots, x_{T}\right\}$.

The statistical features include: \#wlen $n_{n}$ - the number of DNS names with $n$ labels, \#wlen $n_{i, j}$ - the number of labels of the $i^{\text {th }}$ level (with $i \in L$ ) having $j$ characters, \# firstchar $_{i, j}$ - the number of labels at the $i^{\text {th }}$ level (with $i \in L$ ) 
starting with character $j \in C$ and \#ngram $i, j, k$ - the number of times that a character $j \in C$ is succeeded by $k \in C$ at the $i^{\text {th }}$ level with $i \in L$.

These features are transformed into distributions as follows ((2) in figure 1):

- the distribution for domain lengths (in label levels):

$$
\operatorname{distwlen}(X=j)=\frac{\# \text { wlen }}{\sum_{k} \# \text { wen }}
$$

- the distribution of the lengths of labels (in number of characters) for a given level $l$ :

$$
\operatorname{distwlen}_{l}(X=j)=\frac{\# \text { wlen }_{l, j}}{\sum_{k} \# \text { wlen }_{l, k}}
$$

- the distribution of the first characters of labels for a given level $l$ :

$$
\operatorname{distfirstchar~}_{l}(X=j)=\frac{\# \text { firstchar }_{l, j}}{\sum_{k} \# \text { firstchar }_{l, k}}
$$

- the N-gram distribution for a certain level $l$ and a character $c$ is given by:

$$
\operatorname{ngram}_{l, c}(X=i)=\frac{\# n \operatorname{mram}_{l, c, i}}{\sum_{k} \operatorname{ngram}_{l, c, k}}
$$

N-gram Model: N-grams [14 are successive character sequences of length $n$ $\in \mathbb{N}$, extracted from a string. For example, an n-gram with $n=2$ is called bigram. Consider the following DNS name, test.uni.lu, bigrams can be: te, es, st, un, ni, lu... For generating the names of labels, the different estimated distributions are applied to a Markov chain. A Markov chain is defined for each label level, $l$, as a set of states $S=\left\{s_{1}, s_{2}, \ldots, s_{r}\right\}$ representing the characters that have been observed at this level. The probability of the transition between the two nodes representing by the two characters $c_{i}$ and $c_{j}$ is equivalent to $\operatorname{gram}_{l, c_{i}}\left(X=c_{j}\right)$. By applying $k$ steps, this model allows to generate a label of $k$ characters.

An example for the n-gram model Markov chain is given in figure 2. This means, the probability that a character ' $u$ ' is followed by character ' $n$ ' is 0.4 and the probability that an ' $\mathrm{i}$ ' is followed by another ' $\mathrm{i}$ ' is only 0.2 .

Name Generation: Once the system is trained, SDBF can generate new names to probe, by first defining how long the new name should be in terms of number of labels. To achieve this, a random number following the distribution of number of labels, (distwlen), is generated. As SDBF is designed to be highly customizable, this value can also be set by the user. The same process is applied to determine the length of labels in characters for each label $l$ to generate: distwlen $l$. Again, the user can set the value. Finally, for a label with a length $k$, the first character will be generated following the distribution of the first characters corresponding to the label level, distfirstchar ${ }_{l}$, and the remaining $k-1$ characters are generated by applying the Markov Chain. 


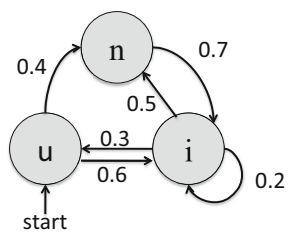

Fig. 2. Markov chain for n-grams

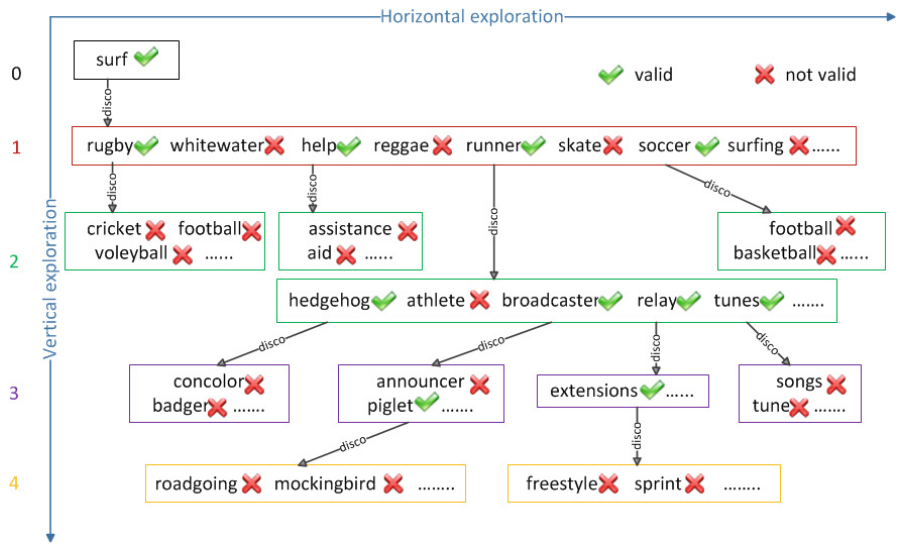

Fig. 3. Example semantic exploration from surf .apple.com

As the Markov chain is limited to a fixed set of transition, some transitions are not possible. For instance, if the bigram "sn" was never observed, the word "snt" could not be generated. To strengthen the discovery, we consider that a transition between any two pairs of characters is possible with a probility $\epsilon$. For this, the other tansitions probabilities are slightly decreased to keep the sum of probabilities for outgoing transitions equal to one.

Because it is common usage to scan a domain, the user can set fixed parts for a domain. For example, the objective may be fixed to discover all domains following the *. uni.lu or ns.*.lu or www.*.*.

Once names are generated, (3) in figure 1, their existence is checked by the name checker (4), which makes a DNS query. This formally corresponds to a function $\operatorname{valid}(D)$ returning the valid domains of a set $D$.

\section{Semantic Extension}

As illustrated in figure 1, the semantic module takes as input a list of names, where the validity has been checked (5). The goal is to extend this list of discovered names by analyzing individual labels. There are two modules, DISCO and the incremental module that can be used individually or combined together, whereas the splitter module is an optional preprocessing step.

\subsection{Similar Names}

The first semantic extension aims to discover names that are similar or related. These are distinct notions 4]. Similarity refers to words having a close meaning (for example, notebook and laptop). Semantic relatedness refers to words sharing the same semantic field like mars and venus, which are different planets. 
As claimed by Kilgraff et. al [11, these usual notions impliy a manual analysis to establish relationships between words that limits its applicability and its extension to further language or semantic domain.

In this paper, we leverage DISCO (extracting DIStributionally related words using CO-occurrences) [12, which is based on an efficient and accurate method for approximating automatically (based on leanring samples) these two notions within one metric, called the similarity afterwards. DISCO considers the distance between two words within a window by defining $\left\|w, r, w^{\prime}\right\|$, the number of times the word $w^{\prime}$ occur after $r$ words after the word $w$, where $-3 \leq r \leq 3$. For example, table 1 represents windows centered on services. The window is moving along all the database samples to compute the counting that is transformed into frequencies, i.e. $f\left(w, r, w^{\prime}\right)$, by dividing by the total number of counted co-occurences for any $\left\|w, r, w^{\prime}\right\|$.

Intuitively, two words $w_{1}$ and $w_{2}$ are considered similar, if both of them have many co-occurrences with the same words, in particular, if the positions the latter regarding $w_{1}$ and $w_{2}$ are similar. DISCO uses the following definition, initially proposed in 13 :

$$
\operatorname{sim}\left(w_{1}, w_{2}\right)=\frac{\sum_{(r, w) \in T\left(w_{1}\right) \cap T\left(w_{2}\right)} I\left(w_{1}, r, w\right)+I(w 2, r, w)}{\sum_{(r, w) \in T\left(w_{1}\right)} I\left(w_{1}, r, w\right)+\sum_{(r, w) \in T\left(w_{2}\right)} I\left(w_{2}, r, w\right)}
$$

where $I\left(w, r, w^{\prime}\right)$ is the mutual information between $w$ and $w^{\prime}$ [9] and $T(w)$ all the pairs $\left(r, w^{\prime}\right)$ where $I\left(w, r, w^{\prime}\right)$ is positive.

Assuming a domain $d$ including the label $l$, the objective is to find similar labels $l^{\prime}$. The exploration goes into two directions. The first one is the horizontal exploration, which may be adjusted by $\lim _{h}$. This corresponds to select the most $\lim _{h}$ similar words from DISCO. This result is set into a new set of labels $\operatorname{Expl}_{H}\left(l, \lim _{h}\right)$ which are tested by the Name Checker (figure 1) by concatenating with unmodified labels (other levels). By this, a new set is obtained, denoted by $\operatorname{Valid}\left(\operatorname{Expl}_{H}\left(l, \lim _{h}\right)\right)$.

The second exploration examines the vertical dimension by looking for additional similar names starting from this new set. The limit of the vertical

Table 1. Example of co-occurrence counting (2 windows centered on services)

\begin{tabular}{|c|c|c|c|c|}
\hline \multirow{2}{*}{\begin{tabular}{|c|} 
position \\
sample 1 \\
\end{tabular}} & & -2 & -1 & $+1+2$ \\
\hline & $\mathrm{a}$ & client & uses & services of the platform \\
\hline \multicolumn{5}{|c|}{\begin{tabular}{|l|l|} 
sample 2 & the platform provides services to the client \\
\end{tabular}} \\
\hline \multicolumn{3}{|c|}{$\begin{array}{l}\| \text { services },-3, a \|=1 \\
\| \text { services },-2, \text { client } \|=1 \\
\| \text { services },-1, \text { uses } \|=1 \\
\| \text { services }, 1, \text { of } \|=1 \\
\| \text { services }, \mathbf{2}, \text { the } \|=\mathbf{2} \\
\| \text { services } 3 \text {, platform } \|=1\end{array}$} & \multicolumn{2}{|r|}{$\begin{array}{l}\| \text { | } 2 \text { services },-3, \text { the } \|=1 \\
\| \text { services },-2, \text { platform } \|=1 \\
\| \text { services },-1, \text { provides } \|=1 \\
\| \text { services }, 1, \text { to } \|=1 \\
\| \text { services }, 3, \text { client } \|=1\end{array}$} \\
\hline
\end{tabular}


exploration is set by $\lim _{v}$ and is defined by repeating the previous process $\lim _{v}$ times with new discovered valid names:

$$
\operatorname{Expl}_{V}\left(l, \lim _{v}\right)= \begin{cases}\emptyset & \text { if } \lim _{v}=0 \\ \bigcup_{l^{\prime} \in \operatorname{Expl}_{H}\left(l, \lim _{h}\right)} \operatorname{Valid}\left(\operatorname{Expl}_{H}\left(l^{\prime}, \lim _{H}\right)\right) & \text { if } \lim _{v}=1 \\ \bigcup_{l^{\prime} \in \operatorname{Expl}_{V}\left(l, \lim _{v}-1\right)} \operatorname{Valid}\left(\operatorname{Expl}_{H}\left(l^{\prime}, \lim _{H}\right)\right) & \text { otherwise }\end{cases}
$$

In order to reduce the search space only validated labels are considered for further extensions, as noticed by the use of Valid in the equation (6). The vertical exploration stops once no new correct labels are found. So, $\lim _{v}$ does not need to be manually set, which improves the easy use of our tool.

The vertical exploration is actually recursive and highlighted in figure 1 by the loop (5)-(6)-(7)-(8). Figure 3 represents a subset of a real probing by starting from the label surf, the horizontal exploration reveals unsuccessful (surfing, skate) and successful (rugby, soccer...) labels. Then, the vertical exploration entails a horizontal extension for each of the latter.

\subsection{Incremental Discovery}

In many cases, machines and services are replicated and/or respect a systematic naming scheme as for example pc1, pc2, etc. Assuming that one of them has been discovered, the others can be generated by finding out the numerical components and using the following heuristic: test all possible values (including $\oslash$ ) for each individual digit. This limits the exploration to a number of the same or of smaller power of ten ( 0 to 9 will in the previous example). Preliminary experiments have shown that increasing the search range to bigger numbers does not improve the results while, the overhead highly increases.

\section{$4.3 \quad$ Splitter}

Labels of DNS names can be composed of several words like linuxserver or linux-server. Applying DISCO on such names cannot provide any results since it performs over single words. Therefore, the labels have to be divided automatically in advance. Using a list of separating characters, as for instance "-" is too restricted and our tools refer to the word segmentation method described in [20]. The process is recursive by successively dividing the label in 2 parts, to find the best combination, i.e. with the maximum probability, of the first word and the remaining part. Therefore, a label $l$ is divided in 2 parts for each position $i$ and the probability is computed:

$$
P(l, i)=P_{w o r d}(\operatorname{pre}(l, i)) P(\operatorname{post}(l, i))
$$

where pre $(l, i)$ returns the substring of $l$ composed of the first $i$ characters and $s u b(l, i)$ of the remaining part. $P_{w o r d}(w)$ returns the probability of having the word $W$ equivalent to its frequency in a database of text samples. 
Additionally, the splitter modules can also discover the incremental part of a domain. A label like computer23 is split as computer and 23 which is helpful for the first step of the incremental process (see previous subsection). This may also detect non numerical increments, as observed in our database (servera, serverb, etc.), which can be incremented afterwards using ASCII codes.

\section{Evaluation}

\subsection{Methodology}

Assuming a domain $d$, dictionary based techniques probe by iterating over a set of labels, $l$, to form the hostname $l . d$. In the current evaluation, SDBF is configured similarly and two dictionary-based tools are also tested: Fierce 1 and DNSenum2. Both are included in Backtrack [1, a Linux distribution designed for digital forensics and penetration testing. The dictionary from Fierce includes only 1895 words, whereas the one from DNSenum includes 266930 entries. Hence, SDBF was configured to generate as many labels as DNSenum. The reader should refer to [21] for an evaluation of these tools without semantic extension. The main result is that SDBF and Fierce provide the best results, but all of them are complementary, i.e. they do not find the same names.

Based on the discovered names, new ones are probed using the semantic extensions with one of the following strategies:

- Similar names (DISCO)

- Similar names (DISCO) + Splitter

- Similar names (DISCO) + Splitter + Incremental discovery

Except if mentioned, the last one is applied. The original databases provided with the semantic tools 1220 , like Wikipedia 3 , are used to train them.

The targeted domains in our experiment are extracted from the top 50 websites ranked by Alexa (www.alexa.com), where only 19 domains have been selected such as google.com, ebay.com, baidu.com... This selection discards domains performing wildcarding i.e. these domains will always respond positively to DNS requests regardless of the query. Furthermore, similar domains with different TLD have also been discarded, since hostname results are similar in this case. For example, google has no less than twelve domain names with different TLDs in the top 50 Alexa. We also choose five popular domains from Luxembourg including the one of our university (uni.lu) which is probed from internal network. All these domains are presented in figure 5.

\subsection{Main Metrics}

In our experimental evaluation we consider Init $t_{i}$ with $i \in$ $\{S D B F, D N$ Senum, Fierce $\}$, the initial list of discovered domains for each tool and we also define:

\footnotetext{
${ }^{1}$ http://ha.ckers.org/fierce/

2 http://code.google.com/p/dnsenum/

${ }^{3}$ http://www. wikipedia.org
} 


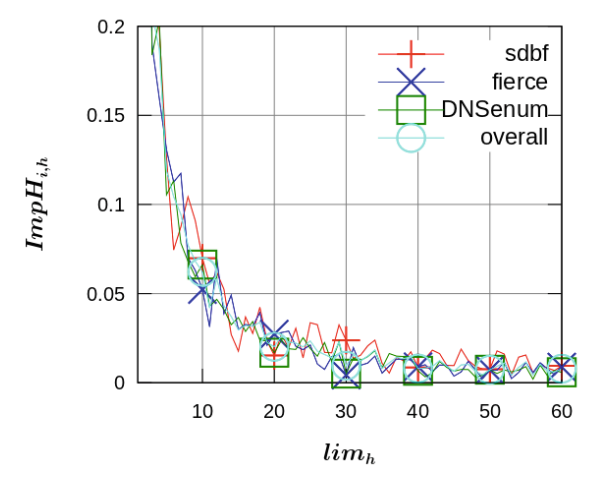

(a) Horizontal exploration

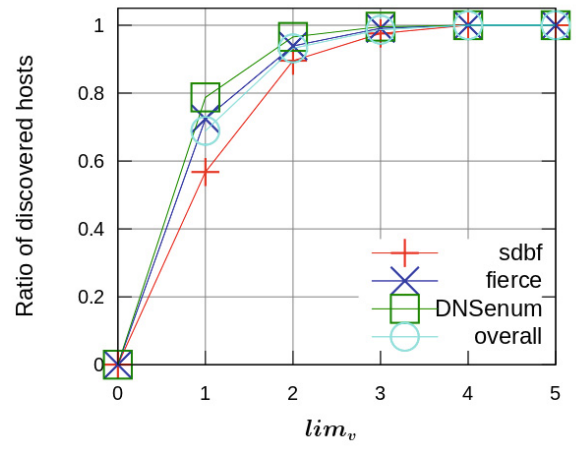

(b) Vertical exploration

Fig. 4. Vertical and horizontal depth analysis (average overall domain)

$$
\text { Init }_{\text {overall }}=\text { Init }_{S D B F} \cup \text { Init }_{\text {DNSenum }} \cup \text { Init }_{\text {Fierce }}
$$

For the evaluation, we present $N e w_{i}$ with $i \in\{S D B F, D N S e n u m$, Fierce, overall $\}$ the set of new discovered domains thanks to every initial dataset Init $_{i}$. Assuming $|S|$ as the cardinality of a set $\mathrm{S}$, the improvement is defined as:

$$
\% \text { Imp }_{i}=\frac{\left|N e w_{i}\right|}{\mid \text { Init }_{i} \mid}, i \in\{S D B F, D N \text { Senum, Fierce, overall }\}
$$

It represents the percentage of new discovered names regarding to the initial dataset. A significant value of $\% \operatorname{Imp} p_{i}$ shows that our method is able to find new hostnames which previous methods have not found, even when they are combined.

\subsection{Exploration Parameters}

Horizontal Search: The horizontal search may be configured by adjusting $\lim _{h}$, which limits the exploration to the top $\lim _{h}$ similar words, as noticed in section 4. On the one hand, we can assume that the more words we have and test, the more hostnames we find. On the other hand, each DNS request is expensive in time and this may lead to the detection of the DNS probe. Figure 4(a) represents the evolution of the hostname discovery regarding $\lim _{h}$, which varies between 1 and 200. The plotted metric, $I m p H_{i, h}$ represents the proportion of new discovered names when $\lim _{h}=h$ compared to $\lim _{h}-1$. Assuming $\% I m p_{i, h}$, the value of $\% I m p_{i}$ when $\lim _{h}=h$, we define:

$$
I m p H_{i, h}= \begin{cases}\% I m p_{i, h} & \text { if } h=1 \\ \% I m p_{i, h}-\% I m p_{i, h-1} & \text { otherwise }\end{cases}
$$

Figure 4(a) shows that having an exploration limit higher than 40 words does not significantly improve the results. That is why we set $l i m_{h}$ to 40 but, in case 


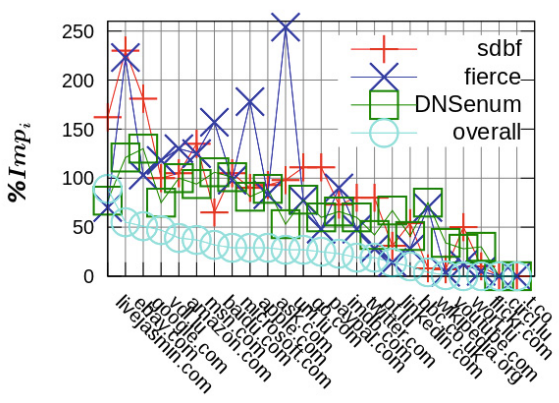

Domain name

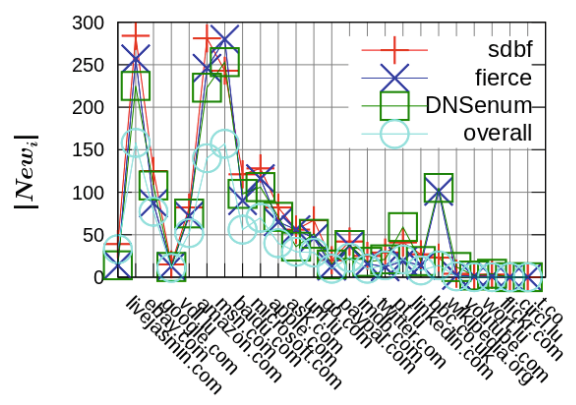

Domain name

(a) Percentage $\left(\% I m p_{i}\right)$ of newly discov- (b) Number $\left(\left|N e w_{i}\right|\right)$ of newly discovered ered hostnames hostnames

Fig. 5. Efficiency of semantic exploration

a deep domain investigation is required, by increasing it, it can still discover new names, as the curves are still positive. Besides, performances are equivalent, whatever the initial tool is.

Vertical Search: As our probing method is based on previous discovered hostnames, we can launch it over new hostnames, gathered by the different process iterations. This number of performed probes is called the vertical depth and fixed through $\lim _{v}$. The process also stops once no new generated names are valid (see section 4). In our case, this leads to a maximal number of 5 iterations. Figure 4(b) represents the ratio of discovered names compared to the maximum $\left(\lim _{V}=5\right)$. Between 55 and $80 \%$ of the domain names are found in the first iteration and more than $95 \%$ before the fourth one, so we can reasonably limit the probe to three iterations.

\subsection{Gain Evaluation}

Figure 5 shows the result of our probe, made on 24 domain names using DISCO with the previously tuned parameters. Regarding the individual improvements, in many cases the number of discovered hostnames is doubled $(\% \mathrm{imp}>100)$ or even more. For instance with the original dataset from SDBF, the number of names related to domains, as go.com, msn.com or google.com, is increased by more than $100 \%$, moreover for ebay.com, we reach an improvement of more than $200 \%$ for both, SDBF- and Fierce-based intialization. Similar results can be observed for DNSenum and the mean improvement over the 24 domains is between $84 \%$ and $102 \%$ as shown in Table 2 .

Furthermore, this tool provides a real solution to discover new hostnames that existing solutions are unable to find, even if all the three other tools are combined (overall in table 2). For instance, a global improvement of $55 \%$ for ebay.com, $51 \%$ for google.com or $30 \%$ on the overall domains set is observed. 
Table 2. Probing results - top 10 and over all domains

\begin{tabular}{|c|c|c|c|c|c|c|c|c|c|c|c|c|}
\cline { 2 - 16 } \multicolumn{1}{c|}{} & \multicolumn{3}{c|}{ SDBF } & \multicolumn{3}{|c|}{ Fierce } & \multicolumn{3}{c|}{ DNSenum } & \multicolumn{3}{c|}{ Overall } \\
\hline Domains & $\mid$ Init $\mid$ & $\mid$ New $\mid$ & $\%$ Imp & $\mid$ Init $\mid$ & New $\mid$ & $\%$ Imp & Init $\mid$ & New & $\%$ Imp & Init $\mid$ & $\mid$ New & $\%$ Imp \\
\hline \hline livejasmin.com & 24 & 39 & 162 & 20 & 14 & 70 & 18 & 14 & 77 & 37 & 33 & 89 \\
\hline ebay.com & 123 & 284 & 230 & 115 & 257 & 223 & 185 & 225 & 121 & 284 & 158 & 55 \\
\hline google.com & 69 & 125 & 181 & 84 & 87 & 103 & 83 & 108 & 130 & 149 & 77 & 51 \\
\hline vdl.lu & 15 & 15 & 100 & 11 & 13 & 118 & 16 & 12 & 75 & 23 & 11 & 47 \\
\hline amazon.com & 78 & 82 & 105 & 55 & 72 & 130 & 75 & 75 & 100 & 132 & 52 & 39 \\
\hline msn.com & 207 & 281 & 135 & 196 & 246 & 125 & 236 & 223 & 94 & 372 & 140 & 37 \\
\hline baidu.com & 369 & 243 & 65 & 178 & 280 & 157 & 238 & 253 & 106 & 478 & 157 & 32 \\
\hline microsoft.com & 115 & 121 & 105 & 91 & 90 & 98 & 97 & 98 & 101 & 189 & 56 & 29 \\
\hline apple.com & 141 & 128 & 90 & 65 & 116 & 178 & 130 & 106 & 81 & 241 & 70 & 29 \\
\hline ask.com & 88 & 82 & 93 & 78 & 65 & 83 & 79 & 71 & 89 & 135 & 40 & 29 \\
\hline \hline all domains & 2057 & 1739 & 84 & 1520 & 1558 & 102 & 1788 & 1565 & 87 & 3170 & 954 & 30 \\
\hline
\end{tabular}

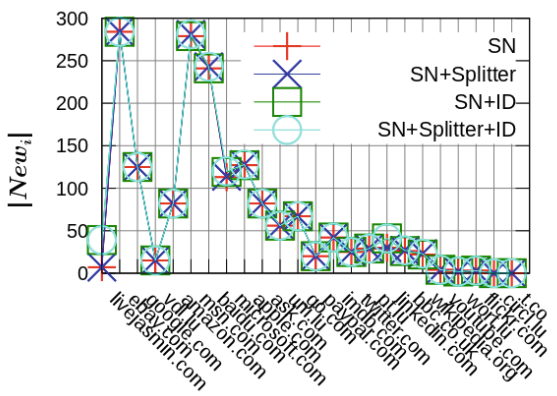

Domain name

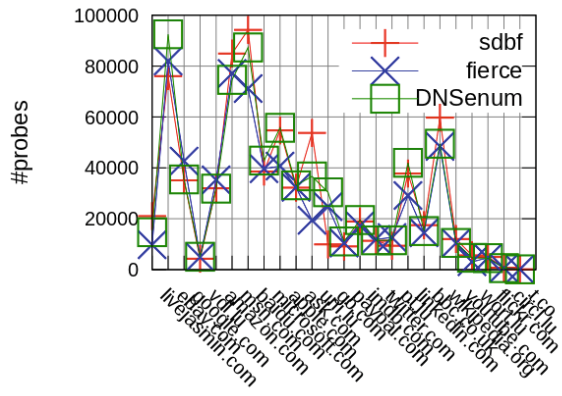

Domain name

Fig. 6. Efficiency of the different semantic Fig. 7. Number of probes per domain to extension when initialized with SDBF discover $\left|N e w_{i}\right|$

This proves the usefulness and accuracy of semantic exploration as the most common hostnames have already been discovered by one of the initial tools (SDBF, Fierce or DNSenum). From a domain name such as mars.pt.lu, merkur.pt.lu and jupiter.pt.lu have been found or from kangaroo.apple. com, we discover camel. apple.com, porcupine.apple.com and piglet.apple. com. Our first assumption deduced from observations that hostnames are attributed by human and by this, a semantic relation exists between hostnames, proves correct.

\subsection{Strategy Evaluation}

As introduced in section 5.1, different strategies are tested by combing DISCO (SN - similar names), the splitter and the incremental modules. Figure 6] shows the efficiency of each startegy initialized with SDBF. We clearly see that Similar Names leads to discover the main part of new DNS names, as curves of other strategies mainly coincide with the one from Similar Names. 


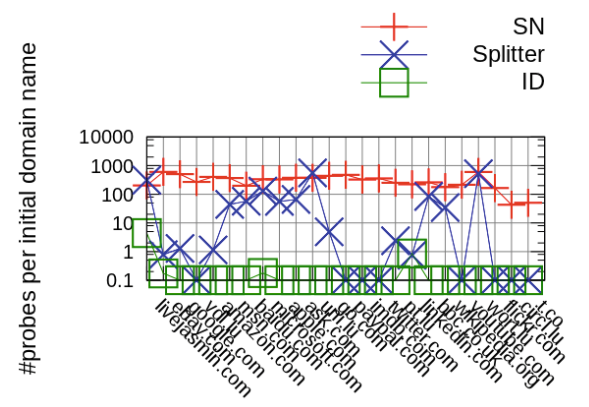

Domain name

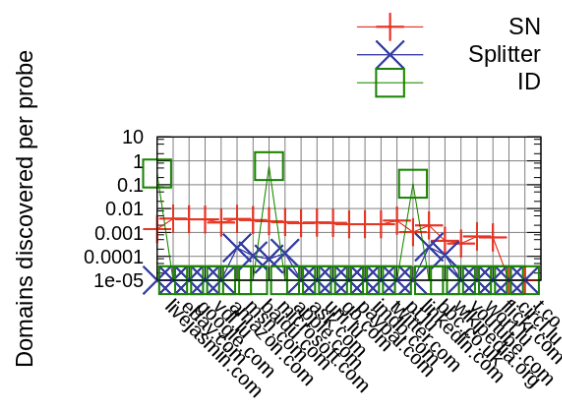

Domain name

(a) Number of probes made per domain in (b) Number of newly discovered hostthe initial dataset names per probe

Fig. 8. Ratio of probes due to each individual module

The second observation is that Splitter provides few signs of improvement to Similar Names and Incremental Discovery (ID) brings some results, especially for the domain livejasmin.com. In fact through this method, the hostname news10. livejasmin.com leads to discover 31 new hosts (newsX with $X \in$ $\{1 ; 9\} \cup\{11 ; 32\})$.

Therefore, the strategy has to be carefully chosen. For fast probing of many domains, only the DISCO based extension should be used, but if the objective is to probe deeply one domain, all of them have to be combined, since each of them may improve the results.

\subsection{Overhead}

The overhead is defined as the number of additional DNS requests (\#probes). As previously mentioned, SDBF and DNSenum require more than 250000 DNS probes to produce their results. In Figure [7, we can observe that our method always needs to perform less than 100000 DNS requests, but this discovery is based on a list established by a prior tool. The biggest probes are made for the biggest initial datasets (ebay.com, msn.com, baidu.com) but, half of the domains require less than 20000 probes. Figure 8(a) shows that the Similar names module has a quite steady ratio of probes per initial name (between 200 and 500 requests). The efficiency of this module, as we can see in figure $8(\mathrm{~b})$ is also steady, it discovers around 1 domain name for 200 probes. Other modules perform less requests than the previous one, as we can see in figure 8(a) but figure 8(b) shows that applying Splitter is less efficient than Similar names, whereas Incremental discovery needs to perform very few probes to discover new domains. 
These results show that our method is far less expensive than initial ones (at least 4 times for SDBF or DNSenum) for approximatively discovering the same number of domain names (section 5.4). As a basis the Similar names module should be used, which provides the steadiest results although, the efficiency of the other tools is dependent of the targeted domain.

\section{Related Work}

In DNS research, major works deal with the detection of DNS attacks as for example, fast-flux, spamming, anomalies in DNS traces,... and present mots various defensive measures for these threats. Statistical evaluation is used in [2], respectively whitelists and classifiers are referred to, to detect anomalous patterns in RR data for rervealing poisoning attacks. The authors in [3] describe a large-scale passive DNS tool, where features are used to detect anomalies, as for example euclidean distances between entries to identify changes in the lifetimes of domains, etc. In [18, suspicious flux networks are detected by passively capturing DNS traffic. The data evaluation is based on the Jaccard index, similar to [8]. To classify the services, the authors refer to supervised learning, where the C4.5 algorithm is used to separate malicious flux and benign services. In [19], the authors perform analysis and visualization of DNS traffic in different modes, off-line, near-real-time and real-time by combining aggregation to clustering. In [6], the authors show that regular expressions improve filtering capabilities for malicious domain detection and provide more accurate results than black-lists.

In this paper, a more semantic approach is used to explore domains in the Net. Natural language processing (NLP) techniques emerged in the research areas of forensics and security. In [5], an automatic domain name generator is constructed by combining different NLP techniques, as for example by using a syllable to construct new passwords or usernames. A major difference to this work is, in [5] full words are generated. By using different statistical tools, as Kulback-Leibler divergence or Levenshtein edit distances, domain names related to botnets can be detected [24]. In the same context of generating new passwords is the work presented in 23. Here, a new approach relying on probabilistic context-free grammar is used to generate rules in order to crack passwords.

\section{Conclusion}

In this paper, DNS brute forcing tools are enhanced by using semantics, i.e. the average improvement is higher than $80 \%$. When combined with SDBF, the tool only needs a passive DNS database and a set of text samples like Wikipedia. Hence, it may easily be applied and it can be continuously reinforced since the previous databases are continuously evolving. Depending on the context, this paper has assessed the benefit of different strategies, as well as the implied overhead. Future work will deal with distributed probing. 


\section{References}

1. Backtrack linux - penetration testing distribution (accessed on 08/22/11), www. backtrack-linux.org

2. Antonakakis, M., Dagon, D., Luo, X., Perdisci, R., Lee, W., Bellmor, J.: A Centralized Monitoring Infrastructure for Improving DNS Security. In: Jha, S., Sommer, R., Kreibich, C. (eds.) RAID 2010. LNCS, vol. 6307, pp. 18-37. Springer, Heidelberg (2010)

3. Bilge, L., Kirda, E., Kruegel, C., Balduzzi, M.: Exposure: Finding malicious domains using passive dns analysis. In: Network and Distributed System Security Symposium, NDSS (2011)

4. Budanitsky, A., Hirst, G.: Evaluating wordnet-based measures of lexical semantic relatedness. Comput. Linguist. 32 (March 2006)

5. Crawford, H., Aycock, J.: Kwyjibo: automatic domain name generation. Software Practice and Experience 38, 1561-1567 (2008)

6. Dagon, D., Lee, W.: Global internet monitoring using passive dns. In: Proceedings of the 2009 Cybersecurity Applications \& Technology Conference for Homeland Security, pp. 163-168. IEEE Computer Society, Washington, DC (2009)

7. Faltstrom, P., Hoffman, P., Costello, A.: Internationalizing Domain Names in Applications (IDNA). RFC 3490 (Proposed Standard) (March 2003), http://www.ietf.org/rfc/rfc3490.txt, obsoleted by RFCs 5890, 5891

8. Hao, S., Feamster, N., Pandrangi, R.: An internet wide view into DNS lookup patterns. Tech. rep., School of Computer Science, Georgia Tech (June 2010)

9. Hindle, D.: Noun classification from predicate-argument structures. In: 28th Annual Meeting on Association for Computational Linguistics, ACL. Association for Computational Linguistics (1990)

10. Kamra, A., Feng, H., Misra, V., Keromytis, A.: The effect of dns delays on worm propagation in an ipv6 internet. In: Proceedings of IEEE Infocom. IEEE, Miami (2005)

11. Kilgarriff, A.: Thesauruses for natural language processing. In: Natural Language Processing and Knowledge Engineering (October 2003)

12. Kolb, P.: Experiments on the difference between semantic similarity and relatedness. In: 17th Nordic Conference of Computational Linguistics NODALIDA. Northern European Association for Language Technology (2009)

13. Lin, D.: Automatic retrieval and clustering of similar words. In: 17th International Conference on Computational Linguistics - COLING. Association for Computational Linguistics (1998)

14. Manning, C.D., Schütze, H.: Foundations of Statistical Natural Language Processing. MIT Press, Cambridge (1999)

15. Mockapetris, P.: Rfc 1035: Domain names - implementation and specification

16. Mockapetris, P.: Rfc 1034: Domain names - concepts and facilities (1987)

17. Mockapetris, P., Dunlap, K.: Development of the domain name system. In: Proceedings of the 1988 ACM SIGCOMM, pp. 123-133. IEEE Computer Society, Stanford (1988)

18. Perdisci, R., Corona, I., Dagon, D., Lee, W.: Detecting malicious flux service networks through passive analysis of recursive dns traces. In: Proceedings of ACSAC 2009, pp. 311-320 (2009)

19. Plonka, D., Barford, P.: Context-aware clustering of dns query traffic. In: Proceedings of the 8th ACM SIGCOMM Conference on Internet Measurement, IMC 2008, pp. 217-230. ACM, New York (2008) 
20. Segaran, T., Hammerbacher, J.: Beautiful Data: The Stories Behind Elegant Data Solutions, ch. 14. O'Reilly Media (2009), http://norvig.com/ngrams/

21. Wagner, C., François, J., State, R., Engel, T., Dulaunoy, A., Wagener, G.: Sdbf: Smart dns brute-forcer. In: To Appear in IEEE/IFIP Network Operations and Management Symposium - NOMS, Miniconference. IEEE Computer Society (2012)

22. Weimer, F.: Passive DNS replication. In: Conference on Computer Security Incident Handling (2005)

23. Weir, M., Aggarwal, S., Medeiros, B.D., Glodek, B.: Password cracking using probabilistic context-free grammars. In: Symposium on Security and Privacy. IEEE

24. Yadav, S., Reddy, A.K.K., Reddy, A.N., Ranjan, S.: Detecting algorithmically generated malicious domain names. In: Proceedings of the 10th Annual Conference on Internet Measurement, IMC 2010, pp. 48-61. ACM, New York (2010) 